\section{Contrôle de l'anxiété par les récepteurs de la sérotonine 5-HT2c de la strie terminale}

\author{
Une explication des effets anxiogènes \\ des inhibiteurs sélectifs de la recapture \\ de la sérotonine (ISRS)?
}

Joël Bockaert, Carine Bécamel
Institut de génomique fonctionnelle, CNRS-UMR5203, Inserm U1191, université de Montpellier, F-34094 Montpellier Cedex 5, France.

joel.bockaert@igf.cnrs.fr
Sérotonine, anxiété et inhibiteurs sélectifs de la recapture de sérotonine La 5-HT (5-hydroxytryptamine ou sérotonine) est impliquée, chez les vertébrés et I'homme, dans un nombre important de fonctions physiologiques et en particulier le contrôle des émotions telles que l'anxiété et les états de l'humeur. Beaucoup de thérapies proposées pour traiter les troubles anxieux - comme les attaques de panique, l'anxiété posttraumatique, les phobies sociales et les troubles obsessionnels compulsifs - ont pour cible la transmission sérotoninergique [1]. Parmi les médicaments les plus prescrits pour traiter ces pathologies se trouvent les fameux inhibiteurs sélectifs de la recapture de sérotonine (ISRS). Le plus connu est la fluoxétine, vendue sous le nom commercial de $\operatorname{Prozac}^{\circledR}$ [2] $(\rightarrow)$.

$\rightarrow$ Voir la Nouvelle de Q. Rainer et al.,$m / s n^{\circ} 10$, octobre 2009, page 795

Les ISRS, en inhibant la recapture de la 5-HT dans la synapse, augmentent sa concentration et donc son efficacité sur les récepteurs sérotoninergiques synaptiques.

Bien qu'efficaces, les traitements par les ISRS posent deux problèmes majeurs. Le premier est que pour obtenir une amélioration des symptômes anxieux, plusieurs semaines de traitements sont nécessaires. Il a cependant été démontré que, dès la prise de ISRS, la concentration synaptique de 5-HT augmente de façon significative. Pourquoi faut-il attendre plusieurs semaines pour obtenir un effet thérapeutique? Plusieurs hypothèses ont été émises mais aucune ne semble entièrement satisfaisante. Le deuxième problème est que, pendant les premières semaines de traitements, les ISRS ont souvent un effet inverse de celui recherché, c'est-à-dire un effet anxiogène [3, 4]. Il est donc important de comprendre les mécanismes moléculaires et cellulaires impliqués dans cet effet précoce non désiré des ISRS.

\section{Récepteurs impliqués dans les effets} anxiogènes paradoxaux des ISRS

Un des tests les plus utilisés pour étudier la peur est le fear conditioning, qui peut être traduit par «conditionnement de peur». Un stimulus neutre (comme un son ou une lumière), appelé stimulus conditionnel ( $S C$ ), est associé une ou plusieurs fois à un stimulus inconditionnel (IS) douloureux (comme un choc électrique) administré quelques secondes après. Cette étape correspond à la phase de conditionnement. La présentation du SC quelques heures ou jours après cette phase va déclencher un réflexe de peur : le freezing ou immobilité de l'animal. Placer le rat ou la souris dans la cage où il a reçu le choc électrique (stimulus contextuel) induit également le freezing. C'est une «mémoire de peur».

Un traitement aigu avec de la fluoxétine augmente l'expression de peur chez les animaux et donc la durée de freezing, ce qui pourrait expliquer les effets anxiogènes des ISRS [4]. Les récepteurs de la sérotonine impliqués dans ce mécanisme sont les récepteurs $5-\mathrm{HT}_{2 \mathrm{C}}{ }^{1}[4,5]$ dont on sait, par ailleurs, qu'un polymorphisme au niveau de leur promoteur est responsable d'une prédisposition au suicide [6]. Ce sont des récepteurs couplés aux protéines $G$ (RCPG) qui stimulent la voie de la phospholipase $C$ et la libération de $\mathrm{Ca}^{+}$intracellulaire, produisant une dépolarisation cellulaire [7]. Il a également été montré que les souris dont le gène codant pour les récepteurs $5-\mathrm{HT}_{2 \mathrm{C}}$ a été invalidé (souris $\mathrm{KO}$, knockout) sont moins anxieuses [8].

\section{L'amygdale et son extension, la strie} terminale, sont impliquées dans l'anxiété

L'amygdale est une des structures cérébrales impliquées dans notre capacité à ressentir et à percevoir certaines émotions dont la peur. Elle reçoit des afférences $^{2}$ de nombreuses régions du système limbique 3 (hippocampe, septum, hypothalamus, cortex frontal et senso-

${ }^{1}$ Les récepteurs de la sérotonine sont codés par 18 gènes, dont 13 codent pour des récepteurs couplés aux protéines $G$ (RCPG), regroupés en 6 familles (récepteurs $5 \mathrm{HTl}, 2,4,5,6$ et 7) [5] en fonction de leur homologie de séquence et leur couplage à la protéine $\mathrm{G}$.

${ }^{2}$ Une afférence est une connexion neuronale provenant d'une structure cérébrale, dont les axones arrivent et font synapse dans la région considérée. Une efférence, au contraire, correspond à une connexion de la région cérébrale considérée vers une autre structure.

${ }^{3}$ Ensemble de structures cérébrales impliquées notamment dans le traitement des émotions. 


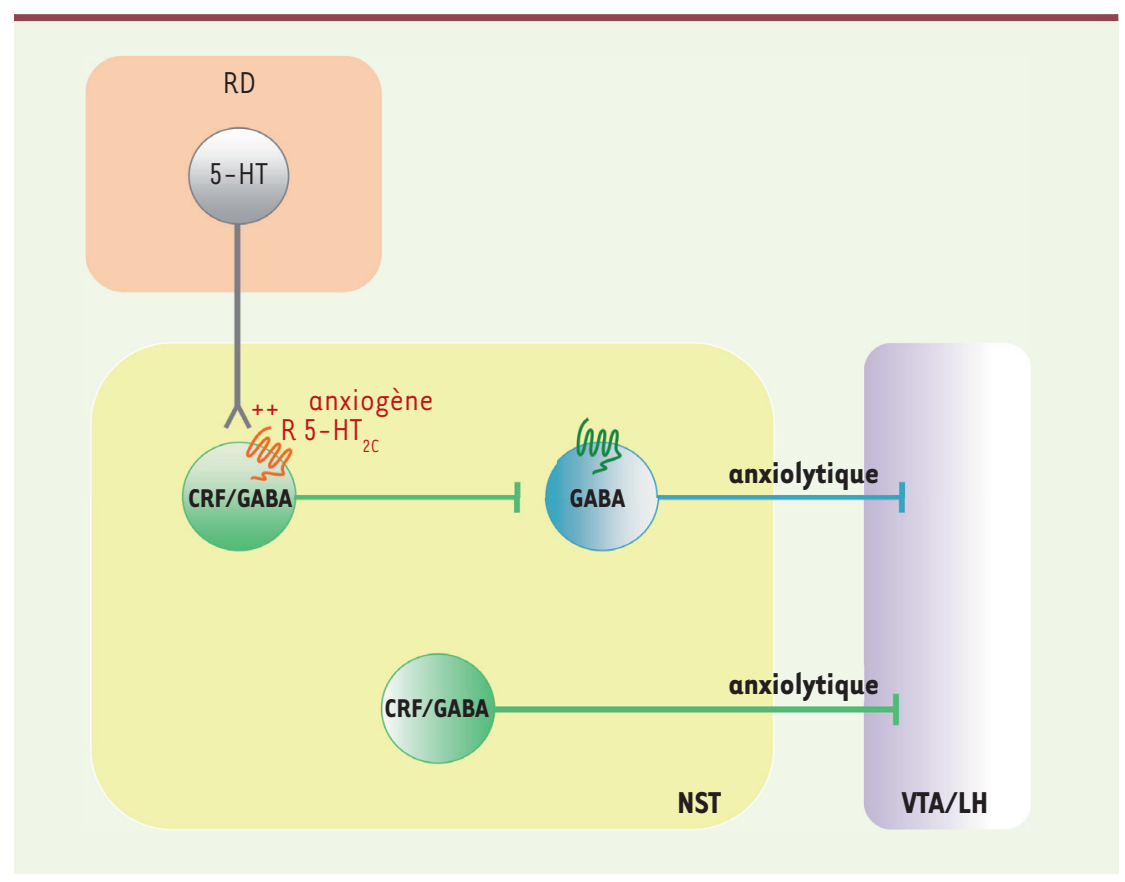

Figure 1. Modèle du microcircuit régulant l'anxiété et l'apprentissage aversif au niveau des noyaux de la strie terminale. Les afférences sérotoninergiques provenant du raphé dorsal (RD) activent les récepteurs $5-\mathrm{HT}_{2 C}$ exprimés au niveau des interneurones CRF/GABA des noyaux de la strie terminale (NST). Ceci entraîne l'inhibition des afférences GABAergiques projetant vers l'aire tegmentale ventrale (VTA) et l'hypothalamus latéral (LH), connues pour être anxiolytiques [10]. La stimulation sérotoninergique favorise donc des comportements anxiogènes (anxiété et peur). II existe également une autre population, plus discrète, de neurones CRF/GABA qui projette directement vers la VTA et le LH et dont l'activation est anxiolytique. 5-HT : sérotonine ; CRF : corticolibérine; GABA : acide $\gamma$-aminobutyrique.

riel, thalamus sensoriel). L'amygdale, activée par ces afférences, va projeter principalement (a) vers le noyau paraventriculaire de l'hypothalamus, ce qui conduira à la libération d'hormone corticotrope $e^{4}$ (ACTH) et donc de cortisol et d'adrénaline par les surrénales, mais aussi (b) vers le locus cœruleus ${ }^{5}$ (activation noradrénergique) et le système sympathique $^{6}$. La plupart des projections provenant de l'amygdale se font via un relai, qui est une extension de l'amygdale appelée la strie terminale [9]. La strie terminale projette aussi vers une

\footnotetext{
${ }^{4}$ Hormone stimulant les glandes surrénales.

${ }^{5}$ Noyau sous-cortical, impliqué notamment dans la production du neurotransmetteur noradrénaline, et jouant un rôle dans les émotions et le sommeil.

${ }^{6}$ Le système sympathique fait partie du système nerveux autonome et contrôle de nombreuses fonctions inconscientes de l'organisme comme la régulation du rythme cardiaque.
}

[11]. Tout un ensemble de techniques modernes a été utilisé pour analyser ces circuits neuronaux: des techniques de traçage des voies nerveuses, de l'optogénétique, I'utilisation de souris génétiquement modifiées (souris knock-out [KO], KO conditionnels, knock-in [KI], manipulations spécifiques de certaines cellules), de l'électrophysiologie, etc. Les auteurs montrent l'existence d'une innervation directe de la strie terminale par les neurones sérotoninergiques du raphé dorsal $^{8}$ (Figure 1). La stimulation par optogénétique des fibres 5 -HT au niveau de la strie terminale induit la libération de 5-HT, un effet augmenté par les ISRS. Cette stimulation ne modifie pas l'acquisition du «conditionnement de peur». En revanche, elle augmente la réponse de peur (freezing) au stimulus conditionnel (SC) et au stimulus contextuel, ainsi que l'anxiété. Ces effets de la 5-HT libérée dans la strie terminale, ou des ISRS, sont bloqués par un antagoniste des récepteurs $5-\mathrm{TH}_{2} \mathrm{C}$, en accord avec la littérature [4]. On peut conclure, à ce stade, que l'activation des récepteurs $5-\mathrm{HT}_{2 \mathrm{C}}$ de la strie terminale induit un effet anxiogène.

Il avait précédemment été montré (a) que les souris invalidées (KO) pour le récepteur $5-\mathrm{HT}_{2 \mathrm{C}}$ sont moins anxieuses, et (b) que les neurones à corticolibérine ${ }^{9}$ (CRF) de la strie terminale ont une activation réduite après un test de conditionnement de peur [8]. Les auteurs ont donc recherché si les récepteurs $5-\mathrm{HT}_{2 \mathrm{C}}$ de la strie terminale étaient localisés sur des neurones CRF [11]. Ils ont ainsi montré qu'il existe deux types de neurones CRF dans la strie terminale : ceux qui projettent directement vers la VTA et l'hypothalamus et ceux qui sont intrinsèques à la structure (les interneurones) [11]. Tous ces neurones sont également GABAergiques (Figure 1). Les récepteurs $5-\mathrm{HT}_{2 \mathrm{C}}$ sont spécifiquement exprimés

\footnotetext{
${ }^{8}$ Noyau sous-cortical, impliqué notamment dans la production de sérotonine, et jouant un rôle dans l'humeur, la douleur et le sommeil.

${ }^{9}$ Neurohormone et neurotransmetteur impliqués par exemple dans la réponse au stress.
} 
par les interneurones CRF/GABA de la strie terminale [11] (Figure I) et favorisent leur activation. Cette activation des interneurones CRF/GABA, suite à celle des récepteurs $5-\mathrm{HT}_{2} \mathrm{C}$ par un agoniste (mCPP, méta-chlorophénylpipérazine) ou par la libération locale de $5-\mathrm{HT}$ (par optogénétique), conduit à l'inhibition des neurones de la strie terminale vers lesquels ils projettent (Figure 1). Il est ainsi possible d'enregistrer des potentiels inhibiteurs (IPSP) dans ces neurones GABAergiques projetant vers la VTA [11]. Or, comme nous l'avons déjà vu, l'activation de ces neurones est anxiolytique [10]. Leur inhibition par les neurones CRF/GABA activés par les récepteurs 5 -HT2c est donc logiquement anxiogène [11] (Figure 1).

Les auteurs ont alors testé si les inhibiteurs des récepteurs CRFI bloquaient les effets anxiogènes des ISRS. L'expérience fut positive [11]. La localisation des récepteurs CRFl impliqués dans cet effet n'est pas encore certaine. Mais ces récepteurs pourraient être exprimés par les neurones GABA projetant vers la VTA car l'augmentation des IPSP, enregistrée dans ces neurones suite à la stimulation par la 5-HT, est bloquée par les antagonistes des récepteurs CRFl [11].

Les antagonistes des récepteurs CRFl pourraient-ils être associés aux ISRS dans les premières semaines d'un traitement contre les anxiétés généralisées et les attaques de panique? Une piste à suivre. $\diamond$

The anxiogenic effects of SSRI are mediated by 5 -HT2c receptors of the stria terminalis

\section{LIENS D'INTÉRÊT}

Les auteurs déclarent n'avoir aucun lien d'intérêt concernant les données publiées dans cet article.

\section{RÉFÉRENCES}

1. Guimarães FS, Zangrossi Jr H, Del Ben CM, Graeff FG. Serotonin in panic and anxiety disorders. In : Müller $\mathrm{CP}$, Jacobs BL, eds. Handbook of the behavioral neurobiology of serotonin. London : Academic Press, $2010: 667-85$.

2. Rainer $Q$, Gardier AM, Hen R, David DJ. Mécanismes des effets comportementaux de type anxiolytique/ antidépresseur de la fluoxétine (Prozac ${ }^{\oplus}$ ) : implication de la neurogenèse hippocampique. Med Sci (Paris) 2009; $25: 795-8$.

3. Gorman JM, Liebowitz MR, Fyer AJ, et al. An open trial of fluoxetine in the treatment of panic attacks. J Clin Psychopharmacol $1987 ; 7$ : 329-32.

4. Burghardt NS, Bush DE, McEwen BS, LeDoux JE. Acute selective serotonin reuptake inhibitors increase conditioned fear expression: blockade with a 5-HT(2C) receptor antagonist. Biol Psychiatry 2007 ; 62: 1111-8.

5. Bockaert J, Claeysen S, Dumuis A, Marin P. Classification and signaling characteristics of $5-\mathrm{HT}$ receptors. In : Müller CP, Jacobs BL, eds. Handbook of the behavioral neurobiology of serotonin. London : Academic Press, 2010 : 103-21.

6. Videtic A, Peternelj $\Pi$, Zupanc T, et al. Promoter and functional polymorphisms of HTR2C and suicide victims. Genes Brain Behav 2009 ; 8 : 541-5.

7. Labasque M, Meffre J, Carrat G, et al. Constitutive activity of serotonin $2 C$ receptors at $G$ proteinindependent signaling: modulation by RNA editing and antidepressants. Mol Pharmacol 2010 ; 78 : 818-26.

8. Heisler LK, Zhou L, Bajwa P, et al. Serotonin 5-HT(2C) receptors regulate anxiety-like behavior. Genes Brain Behav 2007 ; 6 : 491-6.

9. Lebow MA, Chen A. Overshadowed by the amygdala: the bed nucleus of the stria terminalis emerges as key to psychiatric disorders. Mol Psychiatry 2016; 21 : 450-63.

10. Jennings JH, Sparta DR, Stamatakis AM, et al. Distinct extended amygdala circuits for divergent motivational states. Nature $2013 ; 496: 224-8$.

11. Marcinkiewcz CA, Mazzone CM, D’Agostino G, et al. Serotonin engages an anxiety and fear-promoting circuit in the extended amygdala. Nature 2016; 537 : 97-101.
NOUVELLE

Le récepteur des adénovirus
et des coxsackievirus (CAR)
est impliqué dans
les processus cognitifs
Charleine Zussy ${ }^{1,2}$, Fabien Loustalot ${ }^{1,2}$, Felix Junyent ${ }^{1,2}$,
Eric J. Kremer ${ }^{1,2}$, Sara Salinas $^{3}$

\section{La plasticité neuronale}

Lors du développement du système nerveux, les neurones s'organisent en réseaux permettant notre fonctionnement moteur, sensoriel et cognitif. Ces réseaux ne sont cependant pas figés. La plasticité neuronale permet à notre cerveau, via des modifications au niveau synaptique et/ou cellulaire, de s'adapter à l'environnement. La découverte de la génération de nou-

veaux neurones chez l'adulte (processus appelé neurogenèse adulte) [1] $(\rightarrow)$ a provoqué de nombreux débats. II est désormais démontré que ce processus participe à la plas-

$(\rightarrow)$ Voir la Nouvelle de M. Lemasson et P.M. Lledo, $m / s$ $n^{\circ}$ 6-7, juin-juillet 2003, page 664 ticité neuronale. La neurogenèse adulte joue notamment un rôle crucial dans la régulation de nom-
${ }^{1}$ Institut de génétique moléculaire de Montpellier, CNRS 5535, 1919, route de Mende, 34293 Montpellier Cedex 5, France. ${ }^{2}$ Université de Montpellier, 4, boulevard Henry IV, 34967 Montpellier Cedex 2, France. ${ }^{3}$ UMR 1058, Inserm, Université de Montpellier, Établissement français du sang, Pathogenèse et contrôle des infections chroniques, 60 , rue de Navacelles, 34394 Montpellier Cedex 5 , France.

sara.salinas@inserm.fr

breux processus cognitifs comme la mémoire ou dans certains désordres psychiatriques tels que la dépression et le stress post-traumatique [1]. Chez l'être humain, la région sous-granulaire (SGZ, subgranular zone) de I'hippocampe ${ }^{l}$ est

\footnotetext{
${ }^{1}$ L'hippocampe est une structure cérébrale impliquée dans la régulation des processus d'apprentissage et de mémorisation, ainsi que dans la représentation de cartes spatiales.
} 